\title{
MULTISCALE MODELING AND SIMULATION OF A CAHN-LARCHÉ SYSTEM WITH PHASE SEPARATION ON THE MICROSCALE*
}

\author{
L. REISCHMANN ${ }^{\dagger}$ AND M. A. PETER ${ }^{\ddagger}$
}

\begin{abstract}
We consider the process of phase separation of a binary system under the influence of mechanical stress and we derive a mathematical multiscale model, which describes an evolving microstructure taking into account the elastic propertics of the involver materials. Motivated by phase-separation processes observed in lipid monolayers in film-balance experiments, the starting point of the model is the Cahn-Hilliard equation coupled with the equations of lincar clasticity, the so-called ('ahn-Larche system. Owing to the fact that the mechanical deformation takes place on a macrosopic scale whercas the phase scparation happens on a microscopic level, a multiscale approach is impcrative. We assume the pattern of the evolving microstructure to have an intrinsic length scalc associated with it, which. after nondimensionalization, lears to a scaled model involving a smal! parameter $\epsilon>0$, which is suitable for periodic-homogenization techniques. The problem is formally homogenized using the method of two-scalc asymptotic expansions. which lcads to a model of distributed-microstructure type in the limit. Finally, numerical simulations based on finite clements showcasc the model behavior of the distributed-microstructure model.
\end{abstract}

Key words. ("ahn-l arché system, distributed-micrust ructure model, poriodic homogrenization. asymptotic cxpansion, phase scparation

AMS subject classifications. $35 \mathrm{~B} 27,74 \mathrm{Q} 10,35 \mathrm{~B} 36,74 \mathrm{~N} 15$

DOI. $10.1137 / 20 \mathrm{M} 1321103$

1. Introduction. Binary phasc-separation processes are often described by the Cahn-Hilliard equation, a fourth-order equation for an order parameter taking different distiuct values in each of the two phases: see the recent inonograph [22] for cxamplc. It is derived from a free-encrgy potential and ensures mass conservation. The Cahn-Larche system is the result of coupling the Cahn-Ililliard cquation with the equations of linear elasticity in order to take into account mechanical effects in the separation process [16]. The chemical potential of the Cahn-IHilliard cquation is extended by a contribution derived from the clastic energy density and the clasticity tensor depends naturally on the order parameter.

In the classical Cahn-Larché system, all processes are modeled on the same length scalc. However, in certain situations, the phase separation and the mechanics occur on distinctly different scales, which requires a multiscale analysis of the Cahn-Larché system. This can be achieved in a homogenization context by the method of asymptotic expansion and it is the focus of this work.

This study is motivated by the process of phase separation in lipid monolayers, which can be observed in film-balance experiments. where phospholipid monolaycrs are compressed. Such monolayers are investigated in experinents since phosphatidylcholines are the main plosplolipids found in mammalian cell membranes. Tlıc lipid dipalmitoylphosphatidylclıoline (DPPC) is particularly common in this con-

- Reccived by the cditors February 24, 2020: accepted for publication (in revised form) Junc 19, 2020: published clectronically September 23, 2020.

https://doi.org/10.1137/20M1321103

†Institute of Mathematics, University of Augsburg, 86135 Augsburg, Germany (lisa.reischmann( math.uni-augsburg.de).

¥Institute of Mathematics, University of A ugsburg, 86135 Augsburg, Germany and Augshurg Centre for Innovative Technologies. University of Augshurg, 86135 Augsburg. Germany (maltc.peter@ math.uni-augsburg.de, https://appa.math.uni-augshurg.de/). 
text (see, c.g., $[13,18,25])$, but many other phosphatidylcholines and, more generally, other phospholipids are used, such as DMPC, DMPG, DMPE, DOPC, DLPC (sce, c.g., $[21,23,14])$. Biomembranes actually have the structure of a bilayer, but monolayers, which provide a sinplified experinental nodel of such membranes, have the advantage of simplified production and, in addition, the molecular density of a monolayer can be controlled by varying the area per molectule on a Langmuir-Blodgett film balance [18]. A film balance is essentially a kind of water trough, equipped with a controllable teflon barrier and a fluorescence microscope. 'The total area of the lipid monolayer and consequently the density of the molecules can be controlled by moving the barrier.

Lipids show different states or phases, corresponding to the alignment of the lipid molecules, depending on certain factors. Gencrally, the lipids in the nonolayer aligu themselves with their hydrophilic head groups in the direction of the water due to their amphiphilic propertics. Assuming constant temperaturc, the monolayer becomes denser and more rigid as the area available for the lipid monolayer is reduced, which corresponds to increasing the lateral pressure. In the liquid-expanded (LE) phase, the molecules are disordered and the chains are partially of convoluted structure. In the liquid condensed (LC) phase, the molecules are much more closely packed and ordered. After spreading the lipid mixture on the film ballance, the lipid molecules initially have plenty of space and are in a state known as the gas-analogue phase. If the space available to the molecules is then reduced, a phase transition occurs in which parts of the monolayer are transferred into the LE phase until, with further compression, the entire monolayer is present in the LE phase. Further compression results in a further phase tramsition into the LC phase. This part is the motivation of this work: In experiments, the formation of two-phase regions can be observed in which regions in the LC phase are dispersed in the less ordered LE phase. The size of the I.C domains is in the range of several unicrons, which differs from the scale of the mechanical deformation induced by the teflon barrier by approximately 5 orders of magnitude. The mechanical processes infuence the size and shape of the arising domains such as specd and strength of compression or waiting periods during multiple compression operations, [18, 13, 25].

By interpreting the lipid monolayer with its coexisting phases as a binary mixture, we use the Calm-Hilliard model, which has already been successfully adapted to study phase-separation processes in lipid bilayers $[4,8]$, to describe the phase separation. In the context of pluase separation in lipid monolayers, the Cahn-Ililliard model has already been extended to take elastic effects into account. In [3], it was coupled with a viscoelastic fluid-flow model to study phase-separation processes in lipid monolayers for a surfarcacoustic-wave-actuated fluid flow for a monolayer in a Huidic regine. Here, we consider the further compressed state. Since the ICC phase of the monolayer is gel-like and has an already relatively densely packed and ordered molecular structure, we interpret the monolayer as a solid and use solid mechanics to describe the mechanical behavior of the monolayer mathematically.

Motivated by these film-balance experiments, in what [ollows, we generally consider a separation process in a binary solid under the infuence of nechanical stress, where the mechanical deformation takes place on a macrosopic scale and the phase separation happens on a microscopic scale. and we use the Cahn-Larche system to model this phenomenon. We note that a (viscoclastic) Cahn-Larché nodel in a multiscale context was used to study the decomposition process in eutectic alloys in [20].

In section 2, we introduce the Cahn-Larché system and discuss it in the filınbalance context. In order to obtain a process-adapted model, in section 3 we pres- 
ent a nondinensionalization, which leads to a scaled system where different involved characteristic length scales are taken into accomnt and which is suitable for periodichomogenization techniques. This system is fornally homogenized in section 4 via two-scale asymptotic expansions. The resulting system is of the so-called distributedmicrostructure type which, in the limit, is a typical result for coupled systems where onc process occurs on the macroscopic scale and the other one on the microscopic scale. Finally, in section 5, nunerical sinulations based on finite elements are presented to showease the model behavior.

2. The Cahn-Larché system. We briefly recall the Cahn-Hilliard model and its extension to the Cahn-Larché model in our notation. For the Cahn-Hilliard model, we define an order parameter $c: \Omega \times(0, T) \rightarrow[0,1]$, which describes the relative concentration of a biuary nixture in a domain $\Omega \subset \mathbb{R}^{N}$ with boundary $\Gamma=\partial \Omega$ over the time interval $(0, T)$, where $T>0$. Pure phases of the components correspond to $c=0$ and $c=1$. We introduce a local frec encrgy density (per volume) $f$ so that the total free energy of the mixture can be defined by

$$
\int_{\Omega} f(c)+\frac{\lambda}{2}|\nabla c|^{2} \mathrm{~d} x
$$

The second term is a regularization term, which penalizes interfaces. The parameter $\lambda$ can be interpreted as related to a line tension (in two dimensions). Specifically, we choose a double-well potential of the form

$$
f(c)=\varphi c^{2}(1-c)^{2},
$$

with a scaling parameter $\varphi>0$. The minimas of the double-well potential are achicved for $c=0$ and $c=1$, i.c., for pure phases. For homogencous mixtures, the local free cnergy is greater. Therefore, it is the driving force for phase separation of a homogeneous mixture.

To arrive at a closed system, we introduce the chenical potential $\mu^{\mathrm{CH}}$, which can be defined via the first variation of the total free energy with respect in $c$, i.c.,

$$
\mu^{\mathrm{CH}}=f^{\prime}(c)+\lambda \Delta c .
$$

According to Fick's law of diffusion, the mass flow is given by

$$
j=-M \nabla \mu^{\mathrm{CH}},
$$

where $M$ denotes the mobility. Conservation of mass leads to an cvolution equation for the relative concentration $c$,

$$
\partial_{i} c=\nabla \cdot\left(M \nabla \mu^{\mathrm{CH}}\right) .
$$

Inserting now the chemical potential defined by (2.3). we obtain the Cahn-Hilliard equation, which describes the change of the relative concentration in tinc in a given domain $\Omega$,

$$
\partial_{t} c=\nabla \cdot\left(M \nabla\left(f^{\prime}(c)-\lambda \Delta c\right)\right) .
$$

For future reference, we note that, according to [15], we choose a constant mobility factor of the form

$$
M=\varphi_{*}^{-1} D
$$


where the dimensional factor $\varphi_{*}$ denotes a cliaracteristic value of the free energy of the system.

The Cahn-Hilliard model does not rapture effects caused by mechanical deformations. This can be achicved by including an clastic contribution in the frec energy, which is the Cahn-Larché model introduced in [16]. If only small deformations are considered. a lincarized theory is applicable. Thus. we consider only infinitesimal strains defined by the linear strain tensor

$$
\mathcal{E}(u)=\frac{1}{2}\left(\nabla u+(\nabla u)^{\mathrm{T}}\right),
$$

where $u: \Omega \times(0, T) \rightarrow \mathbb{R}^{N}$ is the deformation field. Typically, the two phases have different elastic properties. Thus. the elasticity tensor $\mathcal{A}(c)$, which contains the material parameters characterizing the stiffness of the phases, naturally depends on the order parameter $c$. According to Hooke's law, the stress tensor is defined by

$$
\mathcal{S}=\mathcal{A}(c)(\mathcal{E}(u)-\overline{\mathcal{E}}(c)),
$$

where $\overline{\mathcal{E}}(c)$ denotes the eigenstrain. In general, this refers to a strain which is present in the absence of any applied stress. This phenomenon occurs in the presence of inhomogeneities, such as thermal expansions with phase transitions and leads to selfgenerated internal stress [24]. The cigenstrain is often referred to as stress-free strain and, just like the elastic material parameters, it may be different for each phase. A natural choice is a multiple of the identity

$$
\overline{\mathcal{E}}(c)=e(c) \mathbb{1},
$$

where the scalar-valued function $e$ specifies the eigenstrain behavior at a particular phase state and $\mathbb{1} \in \mathbb{R}^{N \times N}$ is the second-order ideutity tensor. So, according to (2.10), the eigenstrain is uniform in all directions, which secms to be a common choice; see, c.g., $[24.7,36]$. According to $[6,12]$ the clastic encrgy density is now given by

$$
\mathcal{W}(u, c)=\frac{1}{2}(\mathcal{E}(u)-\overline{\mathcal{E}}(c)): \mathcal{A}(c)(\mathcal{E}(u)-\overline{\mathcal{E}}(c)) .
$$

Adding the contribution of the clastic cuergy density (2.11) to (2.1), the total cnergy of the system is given by

$$
\int_{S \Omega} f(c)+\frac{\lambda}{2}|\nabla c|^{2}+\mathcal{W}(u, c) \mathrm{d} x
$$

and the chemical potential (2.3) of the system extends to

$$
\mu=f^{\prime}(c)-\lambda \Delta c-\overline{\mathcal{E}}^{\prime}(c): \mathcal{S}+\frac{1}{2}(\mathcal{E}(u)-\overline{\mathcal{E}}(c)): \mathcal{A}^{\prime}(c)(\mathcal{E}(u)-\overline{\mathcal{E}}(c))
$$

Inscrting (2.13) into (2.5) leads to an cxtended Calm-Ililiard cyuation,

$$
\partial_{\ell} c=\nabla \cdot\left(M \nabla\left(f^{\prime}(c)-\lambda \Delta c-\overline{\mathcal{E}}^{\prime}(c): \mathcal{S}+\frac{1}{2}(\mathcal{E}(u)-\overline{\mathcal{E}}(c)): \mathcal{A}^{\prime}(c)(\mathcal{E}(u)-\overline{\mathcal{E}}(c))\right)\right)
$$

This equations needs to be supplemented by an equation of conservation of momentum, which, taking into account (2.9), is given by

$$
\varrho \partial_{t}^{2} u=\nabla \cdot \mathcal{S}=\nabla \cdot(\mathcal{A}(c)(\mathcal{E}(u)-\overline{\mathcal{E}}(c))),
$$


wherc $\varrho$ is the density and neglecting gravitational effects. If we use our representation for the cigenstrain (2.10), then, since

$$
\overline{\mathcal{E}}^{\prime}(c): \mathcal{S}=e^{\prime}(c) \mathbb{1}: \mathcal{S}=e^{\prime}(c) \operatorname{tr}(\mathcal{S}),
$$

we obiain the Cahn-Larché system as follows:

$$
\begin{aligned}
& \partial_{t} c=\nabla \cdot\left(M \nabla \left(f^{\prime}(c)-\lambda \Delta c-e^{\prime}(c) \operatorname{tr}(S)\right.\right. \\
& \left.\left.+\frac{1}{2}(\mathcal{E}(u)-c(c) \mathbb{1}): \mathcal{A}^{\prime}(c)(\mathcal{E}(u)-e(c) \mathbb{1})\right)\right) \quad \text { in } \Omega \times(0, T), \\
& \text { (2.16b) } \varrho \partial_{t}^{2} u=\nabla \cdot(\mathcal{A}(c)(\mathcal{E}(u)-e(c) \mathbb{1})) \\
& \text { in } \Omega \times(0, T) \text {. }
\end{aligned}
$$

The system is completed by initial conditions for $c$ and $u$ and boundary conditions, typically no-flux couditions for $c$ and $\mu$ in order to cusure mass conservation

$$
\nabla c \cdot n=0 \text { on } \Gamma \times S, \quad \nabla \mu \cdot n=0 \text { on } \Gamma \times S,
$$

wherc $n$ denotes the outer normal vector on $\Gamma$. as well as displacenent and/or traction conditions for the displacement.

As noted above. the two phases typically have different elastic properties and hence we denote the clasticity tensor describing the clastic properties of the clastically softer phase by $\mathcal{A}^{\alpha}$ and the elasticity imsor of the elastically stiffer phase by $\mathcal{A}^{\alpha}$. Then, following [36], for the mixture, we consider

$$
\mathcal{A}(c):=\mathcal{A}^{\alpha}+d(c)\left(\mathcal{A}^{\beta}-\mathcal{A}^{\alpha}\right),
$$

all elasticity teusor depending on the relative concentration of the mixture, which is simply an interpolation of the two component tensors. The interpolation function $d$ should be defined such that

$$
d(0)=0, \quad d(1)=1, \quad d^{\prime}(0)=0, \quad d^{\prime}(1)=0,
$$

hence

$$
d(x)= \begin{cases}0, & x<0 \\ -2 x^{3}+3 x^{2}, & 0 \leq x \leq 1 \\ 1, & x>1\end{cases}
$$

is an appropriate choice. With this we have also determined that $c=0$ corresponds to the clastically softer phasc and $c=1$ corresponds to the elastically stiffer phase. We assume positive definiteness for the individual component tensor and the usual symmetry conditions in liucar clasticity theory, i.c., for $\mathcal{A}^{z}=\left(a_{i j k h}^{i}\right)_{1 \leq i . j, k, h \leq N}, i \in$ $\{\alpha, \beta\}$, we require

$$
a_{i j k h}^{i}=a_{i j h k}^{i}=a_{j i k h}^{i}=a_{k h i j}^{i} .
$$

Obviously, the interpolated tensor defined by (2.17) is also positive clefinite and fulfils the symmetry condition (2.19).

Remark 2.1 (cxistence of weak solutions). The existence of solutions of the CahnLarché model is well known. We refer to [9] in particular, where the author studied phase scparation in multicomponent alloys in the presence of clastic interactions and proved the existence of solutions of a mixed formulation of the Cahn-Larché system in a Sobolev-space setting. This solution is shown to be uniquely determined if the clasticity tensor is the sanc for both phases. 


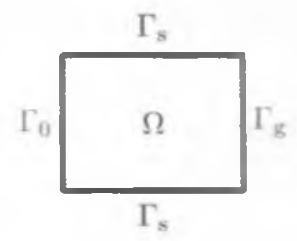

FIG. 1. Domain $\Omega$ with boundary parts $\Gamma_{0}, \Gamma_{\mathrm{g}}$, and $\Gamma_{\mathrm{s}}$.

Remark 2.2 (film-balance setting). For the modeling of phase separation of lipids in a Langmuir-Blodgett film balance. $\Omega \subset \mathbb{R}^{2}$ is a rectangular domain representing the area of the water trough of the film balance on which the lipid nonolayer is examined. and with houndary $\Gamma_{0} \cup \Gamma_{g} \cup \Gamma_{s}$, with boundary parts $\Gamma_{0}, \Gamma_{g}$, and $\Gamma_{s}$ as shown in Figure 1. The force applied by the controllable barrier and by compressing the lipid monolayer is modeled by applying a boundary force $g$ on $\Gamma_{\mathrm{g}}$, hence,

$$
\mathcal{S} n=g \quad \text { onl } \mathrm{r}_{\mathrm{g}} \times S .
$$

On the opposite boundary part $\Gamma_{0}$ we do not expect any deformation and hence we require

$$
u=0 \quad \text { oil } \Gamma_{0} \times S \text {. }
$$

Also, the monolayer camot nove beyond the lateral edges in the normal direction. Therefore, on the side part of the boundary $\Gamma_{\mathrm{s}}$ we set

$$
u \cdot n=0 \quad \text { on } \Gamma_{\mathrm{s}} \times S, \quad n \cdot S \tau=0 \text { on } \Gamma_{\mathrm{s}} \times S,
$$

where $T$ is the tangential vector on $\Gamma$. These conditions describe that the monolayer camnot expand past the lateral edges and does not adhere there when compressed. It would also have been possible to set the free-slip condition on the boundary part $\Gamma_{0}$ as well instead of the Dirichlet condition $u=0$. However, the boundary condition (2.21) cusures the uniqueness of $u$.

3. Nondimensionalization and scaling. In section 1 , we have already pointed out the multiscale aspect of the considered physical process, which assumes the microstructure of the patten to have an intrinsic length scale associated with it. The Cahn-Larché system (2.16a), (2.16b) as introduced in section 2 does not take into account that the processes take place on different scales. A suitable scaling of the system, which takes into account the different characteristic lengths, is therefore necessary; sec, c.g., $[1,11]$ for process-adapted scalings of other systems. In our case, this results naturally from a nondimensionalization, similar to, c.g., $[26,29,28,27,19]$.

To make this more precise, we introducc a characteristic macroscopic length scalc $L$, e.g., representing the order of magnitude of the size of the film balance, and a characteristic microscopic length scale $l$, which corresponds to the order of magnitude of the scale on which the phase separation is observable, and we write

$$
\epsilon=\frac{l}{L} \ll 1
$$

It turus out that the nondimensionalization taking into account the characteristic macroscopic length scalc $L$ and the characteristic microscopic length scale $/$ results in 
a system scaled by powers of $\epsilon$ and which is suitable for the application of techniques of periodic lomogenization.

To facilitate an asymptotic analysis, the order parameter $c$, which describes the microstructure, is assumed to depcnd on two independent spatial variables, $x$. associated with the macroscale and $\frac{x}{r}$, associated with the microscalc. We denote this with an index $r$ and writc

$$
c_{r}(x, t)=c(x, x / \epsilon, t)
$$

Note that we achieve this circumstance in practice by choosing an initial valuc for $c_{e}$, depending on the macroscopic variable $x$ and on the microscopic variable $\frac{x}{e}$. Duc to the dependency on the order parameter, this also applies to the clasticity tensor $\mathcal{A}\left(c_{\epsilon}\right)$, which iuplies au analngue spatial dependence of the displarement field and. hence, we writc $u_{\mathrm{f}}$.

We further define characteristic microscopic lengths associated with the diffusion and the mechanies, $l_{\mathrm{d}}$ and $l_{\mathrm{m}}$, respectively, and express them as a multiple of the geometric microscopic length $l$. Wc choose $l_{\mathrm{l} l}=\frac{1}{10} l$ and $l_{\mathrm{m}}=l$, since the mechanics happens on the whole microscopic length scale whereas the diffusion scale is typirally a little shorter. The characteristic time for the diffusion is then clefined by

$$
T_{\mathrm{d}}:=\frac{l_{\mathrm{d}}^{r} L^{2-r}}{D_{\mathrm{ref}}},
$$

and the characteristic time for the mechanies by

$$
T_{\mathrm{m}}:=\left(\frac{l_{\mathrm{m}}^{\mathrm{s}} L^{2-\mathrm{s}} \varrho_{\mathrm{ref}}}{\sigma_{\mathrm{ref}}}\right)^{1 / 2},
$$

both depending on powers of the two different characteristic length scales. The respective influence of the different characteristic lengths is regulated by exponeuts dopending on parameters $r, s \in[0,2]$, which we have to determine later. With $D_{\text {ref }}, \sigma_{\text {Tef }}$, and $\varrho_{\text {ref }}$ we denote reference values corresponding to the diffusivity, the stiffness. and the density, respectively. Witl the dimensionless macroscopic space variable $\tilde{x}:=x / L$ and the time variable $\vec{t}_{\mathrm{d}}:=t / T_{\mathrm{l}}$ as well as $D=M / \varphi_{\text {. from }}(2.7),(2.16 \mathrm{a})$ becomes

$$
\begin{aligned}
\partial_{i_{\mathrm{d}}} c_{\ell}=\epsilon^{r} 10^{-r} \tilde{\Delta}\left(\tilde{f}^{\prime}\left(c_{\varepsilon}\right)-\epsilon^{2} \bar{\lambda} \bar{\Delta} c-e^{\prime}\left(c_{\varepsilon}\right) \operatorname{tr}(\hat{\mathcal{S}})\right. \\
\left.+\frac{1}{2}\left(\mathcal{E}\left(u_{\varepsilon}\right)-e\left(c_{\varepsilon}\right) \mathbb{1}\right): \tilde{\mathcal{A}}^{\prime}\left(c_{\varepsilon}\right)\left(\mathcal{E}\left(u_{\varepsilon}\right)-e\left(c_{\varepsilon}\right) \mathbb{1}\right)\right),
\end{aligned}
$$

where we have defined the dimensionless quantities as follows:

$$
\tilde{f}^{\prime}\left(c_{\varepsilon}\right):=\varphi_{*}^{-1} f^{\prime}\left(c_{\varepsilon}\right), \quad \tilde{\lambda}:=\varphi_{*}^{-1} \epsilon^{-2} L^{-2} \lambda, \quad \tilde{\mathcal{A}}^{\prime}\left(c_{\varepsilon}\right):=\varphi_{*}^{-1} \mathcal{A}^{\prime}\left(c_{\varepsilon}\right)
$$

and

$$
\tilde{\mathcal{S}}:=\varphi_{*}^{-1} \mathcal{S}=\varphi_{*}^{-1} \mathcal{A}\left(c_{\varepsilon}\right)\left(\mathcal{E}\left(u_{\epsilon}\right)-e\left(c_{\varepsilon}\right) \mathbb{1}\right)=\tilde{\mathcal{A}}\left(c_{\varepsilon}\right)\left(\mathcal{E}\left(u_{\epsilon}\right)-e\left(c_{\varepsilon}\right) \mathbb{1}\right)
$$

with

$$
\tilde{\mathcal{A}}\left(c_{\varepsilon}\right):=\varphi_{*}^{-1} \mathcal{A}\left(c_{\varepsilon}\right)
$$

All quantities with a tilde denote dimensionless quantitics, where $f^{\prime}\left(c_{\varepsilon}\right)$ is of order 1 . Note that the strain is already dimensionless. Further, we have made use of the fact 
that the magnitude of the line tension $\lambda$ is muclı smaller than the free energy level. In order to account for this and to compensate for the length scale associated with the Laplacian. a factor $c^{2}$ is explicitly taken out.

With $\tilde{u}:=\frac{u}{L}$ and with the dimensionless time variable $\bar{t}_{\mathrm{m}}:=t / T_{\mathrm{m}} \cdot(2.16 \mathrm{~b})$ becolnes

$$
\partial_{t_{\mathrm{m}}}^{2} \tilde{u}_{\epsilon}=\epsilon^{s} \tilde{\nabla} \cdot\left(\hat{\mathcal{A}}\left(c_{\varepsilon}\right)\left(\mathcal{E}\left(u_{\varepsilon}\right)-e\left(c_{\kappa}\right) \mathbb{B}\right)\right.
$$

with dimensionless elasticity tensor

$$
\hat{\mathcal{A}}\left(c_{\mathrm{e}}\right)=\sigma_{\mathrm{ref}}^{-1} \mathcal{A}\left(c_{\mathrm{e}}\right) .
$$

Since we want to nondimensionalize the elasticity tensor in a unified way, we put the two different dimensionless variants $(3.8)$ and (3.10) in relation to each other and get

$$
\mathcal{A}\left(c_{\varepsilon}\right)=\sigma_{\text {ref }}^{-1} \mathcal{A}\left(c_{\varepsilon}\right)=\sigma_{\text {ref }}^{-1} \varphi_{*} \overrightarrow{\mathcal{A}}\left(c_{\varepsilon}\right)=\kappa \tilde{\mathcal{A}}\left(c_{\mathrm{e}}\right) \text {. }
$$

In summary, we can state the following dimensionless and scaled system:

$$
\begin{aligned}
& \partial_{\tilde{t}_{\mathrm{d}}} c_{\mathrm{e}}=\epsilon^{\top} 10^{-r} \dot{\Delta}\left(\tilde{f}^{\prime}\left(c_{\ell}\right)-\epsilon^{2} \tilde{\lambda} \tilde{\Delta} c-e^{\prime}\left(c_{\epsilon}\right) \operatorname{tr}(\tilde{\mathcal{S}})\right. \\
& \left.+\frac{1}{2}\left(\mathcal{E}\left(u_{f}\right)-e\left(c_{\epsilon}\right) \mathbb{1}\right): \overline{\mathcal{A}}^{\prime}\left(c_{f}\right)\left(\mathcal{E}\left(u_{\epsilon}\right)-e\left(c_{\epsilon}\right) \mathbb{I}\right)\right), \\
& \partial_{t_{\mathrm{m}}}^{2} \tilde{u}_{r}=\epsilon^{s} \kappa \hat{\nabla} \cdot\left(\tilde{\mathcal{A}}\left(c_{r}\right)\left(\mathcal{E}\left(u_{r}\right)-e\left(c_{\epsilon}\right) \mathbb{1}\right)\right)
\end{aligned}
$$

with a positive dimensionless constant $\kappa:=\sigma_{\text {ref }}^{-1} \varphi_{m}$ that ensures the unification of the dimensionless elasticity tensor in the equations above. The other way around, setting $\overrightarrow{\mathcal{A}}\left(c_{\mathrm{c}}\right)=\kappa^{-1} \mathcal{A}\left(c_{\mathrm{r}}\right)$, of course, could also be chosen.

Special attention must now be paid to the different characteristic times, since they depend on different powers of the scaling parameter $t$. Further, to represcut the diffusion and the mechanics on a common time scale, we need to match the characteristic times and want to unify them as well as possible. Therefore, we require

$$
T_{\mathrm{l}} \approx T_{\mathrm{m}} \approx 1 \text {. }
$$

For a concrete problem, these conditions allow us to determine $r$ and $s$. In what follows, we take the extrenc cases $r=2$ and $s=0$, which correspond to the mechanics being associated with the macroscale and the phase separation associated with the microscale. We show how these numbers arise in the case of the film-balance experiments in Remark 3.1 below.

Dropping the tildes for a simpler notation and better clarity, we get the following dimensionless and scaled system:

$$
\begin{aligned}
\partial_{t} c_{\epsilon}=\epsilon^{2} 10^{-2} \Delta\left(f^{\prime}\left(c_{\epsilon}\right)\right. & -\epsilon^{2} \lambda \Delta c-e^{\prime}\left(c_{\epsilon}\right) \operatorname{tr}(\mathcal{S}) \\
& \left.+\frac{1}{2}\left(\mathcal{E}\left(u_{\epsilon}\right)-e\left(c_{\epsilon}\right) \mathbb{I}\right): \mathcal{A}^{\prime}\left(c_{\epsilon}\right)\left(\mathcal{E}\left(u_{\epsilon}\right)-e\left(c_{\epsilon}\right) \mathbb{1}\right)\right),
\end{aligned}
$$

$$
\partial_{i}^{2} u_{c}=\kappa \nabla \cdot\left(\mathcal{A}\left(c_{\varepsilon}\right)\left(\mathcal{E}\left(u_{\iota}\right)-e\left(c_{\iota}\right) \mathbb{1}\right)\right) .
$$

This systen takes the different scales into accuunt by its scaling by expunents of 6 . Note that so far no assumption has been made about the periodicity of the microstructure, but only about the existence of macroscopic and microscopic characteristic lengths. 
Remark 3.1. For the filı-balance setup, we choose $L=1 \mathrm{~m}$ as the charactcristic length corresponding to the macroscopic process and $l=10^{-4} \mathrm{~m}$ as the characteristic microscopic Iength scale. Unfortunately, there are currently no complete parameter sets of measured data from experinents available. Within the context of film-balance experinents, a lipid monolayer seems to be a scusitive system and, in the literature, the values of the physical quantities may vary depending on the specific lipid or lipids. the phase state of the monolayer, temperature, and even on the measuring method. However, we are only interested in the orders of nagnitude to get an approximate estimate of the characteristic times. Considering typical values of the diffusion cocfficient. we choose $1 \mathrm{um}^{2} \mathrm{~s}^{-1}$ as the characteristic value [10]. Hence, we obtain

$$
T_{\mathrm{d}}=\frac{10^{-r} l^{r} L^{2-\tau}}{D_{\mathrm{ref}}}=10^{-5 r+12} .
$$

Assuming that onc DPPC nolecule occupies an arca of approximately $1 / 70$. $10^{-20} \mathrm{~m}^{2}$ in the beginning of the phase transition [35] and with the molar mass of DPPC, which is $734.04 \cdot 10^{-3} \mathrm{~kg} \mathrm{~mol}^{-1}[33]$, we calculate a characteristic value of the order of magnitude $\approx 10^{-2} \mathrm{~kg} \mathrm{~m}^{-2}$ for the density of a lipid monolayer. With $10^{-1} \mathrm{~N} \mathrm{~m}^{-1}$ as the characteristic value of the stiftuess of a lipid monolayer [34], we get.

$$
T_{\mathrm{rn}}=\left(\frac{l^{s} L^{2-s} \varrho_{\mathrm{ref}}}{\sigma_{\mathrm{ref}}}\right)^{1 / 2}=10^{\frac{1}{2}(-4 s-1)},
$$

which, with regard to the definition interval of the parameters, leads to $r=2$ and $s=0$. Note that, with this scaling, we still consider the mechanics too slow compared to the diffusion, which motivates the quasi-st at,ionary sel ling in section 5 .

4. Periodic homogenization via asymptotic expansions. Using the method of two-scalc asyuptotic expansions in the context of periodic homogenization $[2,30$, $11,5]$, we derive an upscaled version of the system $(3.14 a),(3.14 b)$. We assume the unknowns of (3.14a) and (3.14b) to have an asymptotic expansion in $\epsilon$ of the form

$$
c_{\varepsilon}(x, t)=\sum_{i=0}^{\infty} \epsilon^{2} c_{i}(x, x / \epsilon, t) \text { and } u_{c}(x, t)=\sum_{i=0}^{\infty} \epsilon^{i} u_{i}(x, x / \epsilon, t),
$$

whereby the coefficient functions $c_{i}$ and $u_{i}$ are smooth and these as well as their derivatives are $Y$-periodic with respect to the second argument, where $Y$ is the representative unit cell of the microscale. We insert the expansions (4.1) into the scaled Cahn-Larché system (3.14a), $(3.1 \mathrm{bb})$ and identify the coefficients of the different $\mathrm{c}-$ powers. This procedure leads to a cascade of partial differential equalions.

Gencrally, for a function $v=v(x, y)$ depcnding on two variables in the same way as introduced above, we consider $v_{\ell}:=v\left(\cdot, \frac{-}{\epsilon}\right)$, depending only on the variable $x$. Then, the derivative is given by

$$
\frac{\partial}{\partial x_{i}} v_{\epsilon}(x)=\frac{\partial}{\partial x_{i}} v(x, x / \epsilon)+\epsilon^{-1} \frac{\partial}{\partial y_{i}} v(x, x / \epsilon) \text {. }
$$

In what follows, we also write $\nabla_{x}$ and $\nabla_{y}$ for the gradient with respect to $x$ and $y$, respectively.

For reference in the homogenization process, we first introduce a family of periodic boundary-value problems posed on the unit cell $Y$, which are known as the cell problems in linear clasticity. For detailed information on these problems, we refer to 
[5], where the homogenization result for the equations of lincar elasticity was proven using Tartar's method of ascillating test functions. Let $\Omega \subset \mathbb{R}^{N}$ be a bounded domain and $Y=\left(0, l_{1}\right) \times\left(0, l_{2}\right) \times \cdots \times\left(0, l_{N}\right) \subset \mathbb{R}^{N}$ with positive numbers $l_{1}, \ldots, l_{N}$. Let $\mathcal{A}=\mathcal{A}(y)$ with $\mathcal{A}=\left(a_{i j k h_{h}}\right)_{1 \leq i, j, k, h \leq N}$ being an clliptic fourth-order tensor, and $a_{\imath j k h_{h}}$ being $Y$-periodic for $i, j, k, h=1, \ldots, N$. For any $l, m \in\{1, \ldots, N\}$ we consider the vector-valued function $p^{l m}=\left(\psi_{k}^{l m}\right)_{1 \leq k \leq N} \in \mathbb{R}^{N}$ defined by

$$
p_{k}^{l m}(y):=y_{m} \delta_{k l}, \quad y \in Y, \quad k=1 \ldots, N
$$

with $y_{m}$ being the $m$ th component of $y \in Y$. Then. for each $l, m=1, \ldots, N$, we want to find a vector-valued function $\omega^{l m}$ which solves the following ccll problem:

$$
\begin{gathered}
-\nabla_{y} \cdot\left(\mathcal{A} \mathcal{E}_{y}\left(\omega^{l m}\right)\right)=\nabla_{y} \cdot\left(\mathcal{A} \mathcal{E}_{y}\left(p^{l m}\right)\right) \text { in } Y, \\
\omega^{l m} \quad Y \text {-periodic. }
\end{gathered}
$$

According to the notation used so far, we write $\mathcal{E}_{x}$ and $\mathcal{E}_{y}$, where the subscripts indicate the partial derivatives have been taken with respect to the variables $x$ and $y$, respectively.

We now insert the expansions (4.1) into the system (3.14a), (3.14b) and collect the same powers of $\epsilon$. At scaic $\epsilon^{-2}$, provided by the mechanical equation, we obtain

$$
\nabla_{y} \cdot\left(\mathcal{A}\left(c_{0}\right) \mathcal{E}_{y}\left(u_{0}\right)\right)=0 \text { in } \Omega \times Y \times S .
$$

Multiplying this equation by $u_{0}$ and integrating over $Y$ and by parts yiclds

$$
\int_{Y} \mathcal{A}\left(c_{0}\right) \mathcal{E}_{y}\left(u_{0}\right): \mathcal{E}_{y}\left(u_{0}\right) \mathrm{d} y=0
$$

where the boundary integral vanishes due to the $Y$-periodicity of the derivatives of $u_{0}$ and the components of $\mathcal{A}$. Since $\mathcal{A}$ is positive definite, we get

$$
\alpha\left\|\mathcal{E}_{y}\left(u_{0}\right)\right\|^{2} \leq \int_{Y} \mathcal{A}\left(c_{0}\right) \mathcal{E}_{y}\left(u_{0}\right): \mathcal{E}_{y}\left(u_{0}\right) \mathrm{d} y=0
$$

for a constant $\alpha>0$ and which implies $\mathcal{E}_{y}\left(u_{0}\right)=0$ and, hence,

$$
u_{0}=u_{0}(x, t)
$$

depends only on the macroscopic variable $x$ and on time. So, we have found a candidate describing the macroscopic deformation. The $e^{-1}$-term gives

$$
\begin{aligned}
\nabla_{y} \cdot\left(\mathcal{A}\left(c_{0}\right)\left(\mathcal{E}_{x}\left(u_{0}\right)+\mathcal{E}_{y}\left(u_{1}\right)-e\left(c_{0}\right) \mathbb{1}\right)+c_{1} \mathcal{A}^{\prime}\left(c_{0}\right) \mathcal{E}_{y}\left(u_{0}\right)\right) & \\
& +\nabla_{x} \cdot\left(\mathcal{A}\left(c_{0}\right) \mathcal{E}_{y}\left(u_{0}\right)\right)=0
\end{aligned}
$$

which we consider as an equation for the unknown $u_{1}$. Using (4.5) and the $Y$ periodicity of $u_{1}$ we get a well-posed problem

$$
\begin{aligned}
-\nabla_{y} \cdot\left(\mathcal{A}\left(c_{0}\right)\left(\mathcal{E}_{y}\left(u_{1}\right)-e\left(c_{0}\right) \mathbb{1}\right)\right)= & \nabla_{y} \cdot\left(\mathcal{A}\left(c_{0}\right) \mathcal{E}_{x}\left(u_{0}\right)\right) \quad \text { in } \Omega \times Y \times S, \\
& Y \text {-periodic in } y .
\end{aligned}
$$


For further considerations we need to work with the componentwisc represcntation of this equation, namcly,

$$
\begin{aligned}
-\sum_{j=1}^{N} \partial_{y_{j}} \sum_{k, h=1}^{N}\left(a_{i j k h}\left(c_{0}\right)\left(e_{k h y}\left(u_{0}\right)-e\left(c_{0}\right) \delta_{k h}\right)\right) & \\
& =-\sum_{j=1}^{N} \partial_{y_{1}} \sum_{k, h=1}^{N}\left(a_{i j k h}\left(c_{0}\right) e_{k h . \mathrm{I}}\left(u_{0}\right)\right)
\end{aligned}
$$

for $i=1, \ldots, N$. At this step, we want to gain a representation of $\mathcal{E}_{y}\left(u_{1}\right)$ in terms of $\mathcal{E}_{x}\left(u_{0}\right)$. Therefore, we need the auxiliary problems (4.3) for the mechanics, which we also consider now in componentwise form:

$$
-\sum_{j=1}^{N} \partial_{y}, \sum_{k, h_{h}} a_{i j k h}\left(c_{0}\right) e_{k h y}\left(\omega^{l m}\right)=\sum_{j=1}^{N} \partial_{y} \sum_{k, h} a_{i j k h}\left(c_{0}\right) e_{k h x}\left(p^{l m}\right)
$$

for $i=1, \ldots, N$. Recall that the right-hand side is defined as $\left(p^{l \mathrm{rn}}(y)\right)_{k}=y_{m} \delta_{k l}$ for $l, m=1, \ldots, N$. By using the identity

$$
\sum_{k . h=1}^{N} a_{i j k h}\left(c_{0}\right) e_{k h x}\left(p^{l m}\right)=a_{i j l m}
$$

for $i, j, l, m=1, \ldots, N$, we writc $(4.9)$ as

$$
-\sum_{j=1}^{N} \partial_{y_{j}} \sum_{k, h} a_{i j k h}\left(c_{0}\right) e_{k h y}\left(\omega^{l m}\right)=\sum_{j=1}^{N} \partial_{y_{j}} a_{i j l m}\left(c_{0}\right), \quad 1 \leq i \leq N .
$$

Multiplying both sides of (4.11) with $e_{l m x}\left(u_{n}\right)$ and summing up over $l$ and $m$ yields

$$
\begin{aligned}
-\sum_{j=1}^{N} \partial_{y_{j}} \sum_{k, h=1}^{N} a_{i j k h}\left(c_{0}\right) \sum_{l, m=1}^{N} e_{k h y}\left(\omega^{l m}\right) e_{l m x}\left(u_{0}\right) & \\
& =\sum_{j=1}^{N} \partial_{y_{j}} \sum_{l, m=1}^{N} a_{i j l m}\left(c_{0}\right) e_{l m x}\left(u_{0}\right)
\end{aligned}
$$

for $i=1, \ldots, N$. We compare both cquations, namely, (4.12) and (4.8) and from the left-hand sides we can directly read of a representation for $u_{1}$ in terms of $u_{0}$ with the help of the solutions of the cell problems. We obtain

$$
e_{i j y}\left(u_{1}\right)=\sum_{l . m=1}^{N} e_{l m: x}\left(u_{0}\right) e_{i j y}\left(\omega^{l m}\right)+e\left(c_{0}\right) \delta_{i j}, \quad 1 \leq i, j \leq N .
$$


Taking into account that $\mathcal{E}_{y}\left(u_{0}\right)=0$, the $\epsilon^{0}$-term leads to

$\partial_{t} c_{0}=10^{-2} \Delta_{y}\left(f^{\prime}\left(c_{0}\right)-\lambda \Delta_{y} c_{0}-e^{\prime}\left(c_{0}\right) \operatorname{tr}\left[\mathcal{A}\left(c_{0}\right)\left(\mathcal{E}_{x}\left(u_{0}\right)+\mathcal{E}_{y}\left(u_{1}\right)-e\left(c_{0}\right) \mathbb{I}\right)\right]\right.$

$$
\left.+\frac{1}{2}\left(\mathcal{E}_{x}\left(u_{0}\right)+\mathcal{E}_{y}\left(u_{1}\right)-e\left(c_{0}\right) \mathbb{I}\right): \mathcal{A}^{\prime}\left(c_{0}\right)\left(\mathcal{E}_{x}\left(u_{0}\right)+\mathcal{E}_{y}\left(u_{1}\right)-e\left(c_{0}\right) \mathbb{I}\right)\right),
$$

$\partial_{t}^{2} u_{0}=\kappa \nabla_{y} \cdot\left(\mathcal{A}\left(c_{0}\right)\left(\mathcal{E}_{x}\left(u_{1}\right)+\mathcal{E}_{y}\left(u_{2}\right)-e^{\prime}\left(c_{0}\right) c_{1} \mathbb{1}\right)\right.$

(4.14b)

$$
\begin{array}{r}
\left.+c_{1} \mathcal{A}^{\prime}\left(c_{0}\right)\left(\mathcal{E}_{x}\left(u_{0}\right)+\mathcal{E}_{y}\left(u_{1}\right)-e\left(c_{0}\right) \mathbb{1}\right)\right) \\
+\kappa \nabla_{x} \cdot\left(\mathcal{A}\left(c_{0}\right)\left(\mathcal{E}_{x}\left(u_{0}\right)+\mathcal{E}_{y}\left(u_{1}\right)-e\left(c_{0}\right) \mathbb{1}\right)\right) .
\end{array}
$$

Integrating (4.14b) in a componentwise form over $Y$, we obtain

$$
\partial_{i}^{2}\left(u_{0}\right)_{i}=\frac{\kappa}{|Y|} \sum_{j=1}^{N} \partial_{x} \int_{Y} \sum_{k, h=1}^{N} a_{i j k h}\left(c_{0}\right)\left(e_{k h x}\left(u_{0}\right)+e_{k h y}\left(u_{1}\right)-e\left(c_{0}\right) \partial_{k h}\right) d y
$$

for $i=1, \ldots, N$. Thereby, the integral of the first expression of the right-hand side of (4.14b) vanishes due to the $Y$-periodicity of the involved functions. Next, we insert (4.13), the representation for $\mathcal{E}_{y}\left(u_{1}\right)$, into (4.15):

(4.16) $\partial_{t}^{2}\left(u_{0}\right)_{i}=\frac{\kappa}{|Y|} \sum_{j=1}^{N} \partial_{x} \int_{Y^{\prime}} \sum_{l, m, k, h=1}^{N} a_{i j l m}\left(c_{0}\right)\left(\delta_{l k} \delta_{m h}+e_{l n n y}\left(\omega^{k h h}\right)\right) \mathrm{d} y e_{k h . x}\left(u_{0}\right)$

for $i=1, \ldots, N$. In this, we have now found an equation for the macroscopic part of the deformation $u_{0}$. which motivates us to define the effective or homogenized elasticity tensor

$$
\mathcal{A}^{\text {l'om! }}=\left(a_{i j k h}^{\text {horm }}\right)_{1 \leq i, j, k, h \leq N}
$$

by its components

$$
a_{i j k h}^{\text {hom }}:=\frac{1}{|Y|} \int_{Y} \sum_{l, m=1}^{N} a_{i j l m}\left(c_{0}\right)\left(\delta_{l k} \delta_{m h}+e_{l m y}\left(\omega^{k h}\right)\right) \mathrm{d} y
$$

for $i, j, k, h=1, \ldots, N$, and writc (4.16) as a purcly macroscopic equation:

$$
\partial_{\ell}^{2} u_{0}=\kappa \nabla_{x} \cdot\left(\mathcal{A}^{\text {hoin }} \mathcal{E}_{x}\left(u_{0}\right)\right) \text { in } \Omega \text {. }
$$

Furthermore, to use tensor notation for better clarity, we write

$$
\mathcal{E}_{\omega}=\left(e_{l m k h}^{\omega}\right)_{1 \leq L, m, k, h \leq N} \text { with } e_{l m k h}^{\omega}=e_{l m y}\left(\omega^{k h}\right),
$$

and we also use the identity tensor

$$
\mathcal{I}=\left(\mathcal{I}_{l m k h}\right)_{1 \leq l, m, k, h \leq N} \quad \text { with } \quad \mathcal{I}_{l m k h}=\frac{1}{2}\left(\delta_{l m} \delta_{k h}+\delta_{m h} \delta_{l k}\right) .
$$


Now, we can state the formally homogenized system, given by

$$
\begin{aligned}
\partial_{t} c_{0}=10^{-2} \Delta_{y}( & f^{\prime}\left(c_{0}\right)-\lambda \Delta_{y} c_{0}-e^{\prime}\left(c_{0}\right) \operatorname{tr}\left[\mathcal{A}\left(c_{0}\right)\left(\mathcal{I}+\mathcal{E}_{\omega}\right) \mathcal{E}_{x}\left(u_{0}\right)\right] \\
& \left.+\frac{1}{2}\left(\mathcal{I}+\mathcal{E}_{\omega}\right) \mathcal{E}_{x}\left(u_{0}\right): \mathcal{A}^{\prime}\left(c_{0}\right)\left(\mathcal{I}+\mathcal{E}_{\omega}\right) \mathcal{E}_{x}\left(u_{0}\right)\right) \quad \text { in } \Omega \times Y \times S,
\end{aligned}
$$

$$
\partial_{i}^{2} u_{0}=\kappa \nabla_{x} \cdot\left(\mathcal{A}^{\text {hom }} \mathcal{E}_{x}\left(u_{0}\right)\right)
$$

The remaining unknowns of this limit system are $c_{0}$ and $u_{0}$, the coefficient functions of the first terms of the expansions. whereby only $c_{0}$ still depends on the microscopic variable $y$. The system above is of the so-called distributed-microstructure type. In such a model, a unit cell $Y_{x}$ is associated with cach macroscopic point $x \in \Omega$, in which the local (microscopic) cquations are solved, the solutions of which feed into the global (macroscopic) cquation after averaging; cf. [1, 31, 32, 11, 19]. In our linit system the local equation (4.21a) for $c_{0}$ as well as the cell cquations (4.3) have to be solved in every unit cell $Y_{x}$ and the global equation $(4.21 \mathrm{~b})$ for $u_{0}$ has to be solved in \2. On $Y_{x}$, therefore, a microstructure can be secn which is representative near $x \in \Omega$. In particular, the microstructure can evolve differently at cach global point.

Note, that the homogenized tensor depends through $c_{0}$ on both variables, on $x$ and on $y$. Notice further, that the homogenized mechanical cquation does not contain the eigenstrain explicitly. Self-generated tensions on the inicroscale are thus macroscopically averaged out.

We remark that system (4.21) also has an encrgy associated with it given by

$$
\int_{12 \times Y} f\left(c_{0}\right)+\frac{\lambda}{2}\left|\nabla_{y} c_{0}\right|^{2}+\frac{1}{2}\left(\mathcal{I}+\mathcal{E}_{\omega}\right) \mathcal{E}_{x}\left(u_{0}\right): \mathcal{A}\left(c_{0}\right)\left(\mathcal{I}+\mathcal{E}_{\omega}\right) \mathcal{E}_{x}\left(u_{0}\right) \mathrm{d} y \mathrm{~d} x,
$$

which is of similar form to (2.12). Noting that $\mathcal{E}_{\omega}$ depends on $c_{0}$ through (4.13), the gradient-flow structure of the original svstem is inherited by the limit system.

5. Numerical simulations. We want to test the homogenized system phenomenologically and demonstrate what can be realized with the model by two-dimensional simulations. The implenentation was realized using the finite element library FEniCS [17] based on globally continuous, piccewise linear trial and test fuuctions, where the fourth-order equation was taken care of in terms of a mixed finite element method for the concentration and the clicmical potential as an anxiliary rariable. In order to kecp things simple, we take the mechanics as quasi-stationary, i.e., (4.21b) with zero left-hand side; also sce Remark 3.1. For discretization in time of the cxtended Cahn-Hilliard cquation (4.21a), we use a fully implicit Euler scheme, where a Newton method is used to solve the resulting algebraic nonlincar system in cach time step. For comparison, we arcompany the distributed-micrnstructure Cahn-Larché case studies with numerical simulations of the Cahn-Hilliard equation, nondimensionalized corresponding to section 3 , to compare the respective separation process with a separation process without the influence of mechanical stress.

We choose $\Omega=(0,1)^{2}$ as the macroscopic domain provided with the boundary parts as specified in Figure 1 and a standard unit cell $Y_{x_{i}}=Y=(0,1)^{2}$ at each macroscopic point. The macroscopic boundary conditions for the displacement are chosen such that a nommiform macroscopic strain results. For the displacement, we choose a zero Dirichlet boundary condition on three of the four sides of $\Omega$.

$$
u=0 \quad \text { oll }\left(\Gamma_{0} \cup \Gamma_{s}\right) \times S
$$


and compress the domain at the fourth,

$$
u \cdot n=u_{\mathrm{g}} \text { on } \mathrm{I}_{\mathrm{g}} \times S,
$$

where $u_{\mathrm{g}}$ is chosen such that the macroscopic domain is reduced by $5 \%$ in the horizontal direction. A free slip in the tangential direction on $\mathrm{I}_{\mathrm{g}} \times S$ completes the boundary conditions. For $c_{0}$, we have periodic boundary conditions and as initial condition we use a locally randomly perturbed constant function, which is $Y$-periodic in $y$. More preciscly,

$$
c_{\mathrm{in}}(y)=c_{\mathrm{m}}+\xi(y), \quad y \in Y
$$

where $\xi$ is a function drawing random numbers from a uniform distribution in the interval $[-0.005,0.005)$ for each argument and $c_{\text {in }}$ is $Y$-periodic. In what follows, we consider binodal phase separation by choosing $c_{\mathrm{m}}=0.3$ in (5.3) as well as spinodal plıase separation by setting $c_{\mathrm{m}}=0.5$. Otherwise, we always take the exact sane initial value for all simulations and for $c_{0}$ in each $Y_{x_{i}}$. The other parameters are always chosen as $\lambda=10^{-4}$ and $\varphi=0.75$. For the mechanical parameters, we take the Lamé constants

$$
\lambda^{a}=0.6, \quad \mu^{u}=0.6
$$

for the clastically softer phase and

$$
\lambda^{\beta}=1.2, \quad \mu^{\natural}=1.8
$$

for the clastically slightly harder phase, so that

$$
a_{k h l m}^{i}=\lambda^{i} \delta_{k h} \delta_{l m}+\mu^{i}\left(\delta_{k l} \delta_{h m}+\delta_{k+n} \delta_{h l}\right)
$$

for the components of the clasticity tensors $\mathcal{A}^{\prime}, i \in\{a, 3\}$, as well as $e^{\prime}(c)=0.2$ unless stated otherwise. Iu order to realize the micro- macro coupling, at cach node $x_{i}$ of the macroscopic mesh, there is an associated unit cell $Y_{x_{i}}=Y$ provided with a finer mesh compared with the macroscopic one. For a fixed time step size $\tau>0$, which is chosen as $\tau=5 \times 10^{-2}$ in all sinulations, we consider the discrete time steps $t_{k}=t_{k-1}+\tau$ or $t_{k}=k \tau$ for $k=1, \ldots, k_{\max }$ for onc $k_{\text {max }} \in \mathbb{N}$ and we set $T_{\max }:=\tau k_{\max }$. Then, the solution procedure at cach discrete tinc step $t_{k}$ works as follows: In cach tine step, we first solve the cell problems in each unit cell $Y_{x_{i}}$ associatcd with cach macroscopic node $x_{i}$ of the macroscopic mesh. Witl these cell solutions, we assemble and solve the macroscopic equation. 'Then, with the calculated macroscopic displacement, iterating over each macroscopic node $x_{i}$, we solve the cvolution equation for the order parancter in every microscopic cell $Y_{x_{i}}$.

Figure 2 shows a typical result for the magnitude of the displacement with some marked points of the macroscopic donain in which we show the separation processes in the following simulations. Since we are primarily intercsted in the microscopic processes and we do not make any comparisons to experiments, we have only chosen a coarse mesh. The microscopic mesh is chosen in such a way that an alignument of the pattern of the evolving microstructure caused by structures of the mesh is avoided and we refer to [8] for studies ou such mesh effects.

Our choice of boundary conditions for the displacement implies that the strain tensor varies locally and, in particular, includes shear: sec Figure 2. As one can sec 


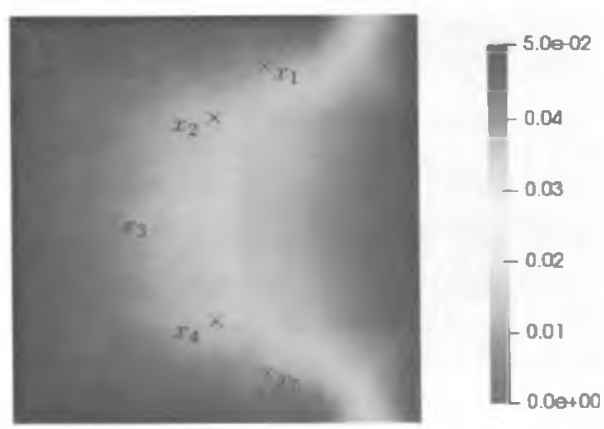

FiG. 2. Typical magnitude of the macroscopic displacement $u_{0}$ and five marked macroscopic points $x_{1}, \ldots, x_{5}$

from (4.21a), the macroscopic strain $\mathcal{E}\left(u_{0}\right)$ enters in the evolution equation describing the evolving inicrostructure and. as it may be different in cach macroscopic point, the nicrostructure is expected to be different in each nacroscopic point in general. In the following simulations, we therefore expect that the phase separations in the different macroscopic points differ from each other and that an influence on the resulting patterns can be detected.

5.1. Binodal phase separation. In the case of binodal plase scparation, we first compare the evolution in time of the separation process described by the distributcd-microstructure moicl in the macroscopic point $x_{1}$ with the separation process without clasticity of the corresponding Cahn-Hilliard simulation. Figure 3 shows the results of the Cahn-Hilliard simulation in the left columm and the results of the simulation of the distributed-microstructure model in the right column. Plots in onc row arc at the same point in time $t_{k}$ specified underneath. For all following simulations of the phasc-scparation processes, we use the color bar given by Figure 4 for the representation of the order parancter $c$. Of course, due to the numerical solution methods and modeling aspects, such as the choice of the local free energy, there may be a deviation beyond the limits 0 and 1 for values of the order parameter. However, these are minimal and for standardization we always use this color bar.

The (standard) Cahn-Hilliard simulations show a typical separation process. Regions of purc phases and domains of onc component (corresponding to $c=1$ ) arc formed in the other contiguous phase (corresponding to $c=0$ ) and the separation process is quite far advanced already at $t_{30}$. As time progresses further, the fusion and growth of the domains can be observed, which corresponds to a reduction of the phase boundaries. The shape of the domains corresponds to the energetically favorable circular shape of the red-colored domains. During the fusion of two domains, cllipsoidal structures also occur temporarily, as can be scen particularly well in Figures $3(\mathrm{~b})$ and $3(\mathrm{~d})$, but these quickly relax again into circularly shaped domains.

Considering the results of the DM simulation (we usc this abbreviation in what follows for the simulation of the distributed-nicrostructure model), one can see that the process is roughly the same as that observed by the Calın-Hilliard simulation. The initially homogeneous mixture separates, regions of pure phases are formed, and the resulting domains of the elastically harder phase merge and grow together. 

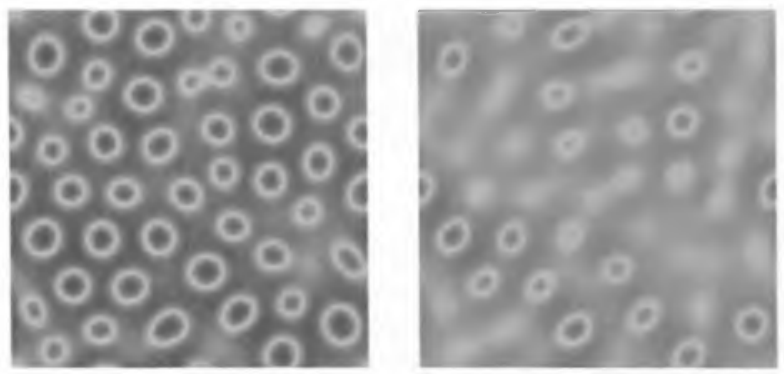

(a) $t_{30}=1.5$
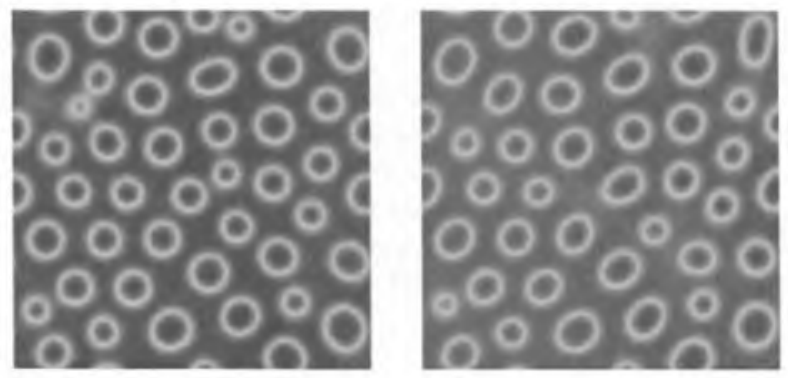

(b) $t_{: 40}=2$
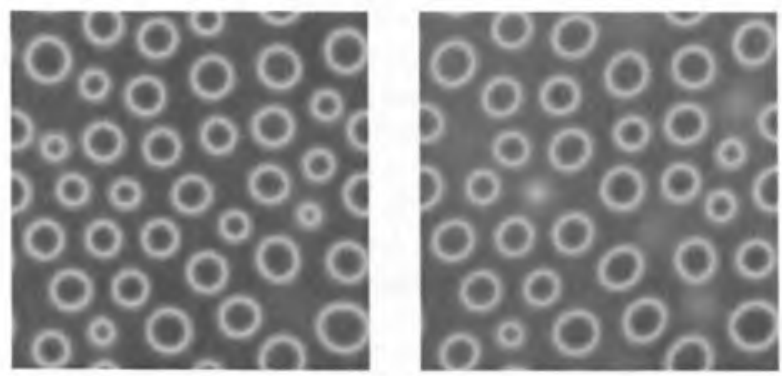

(c) $t_{60}=3$
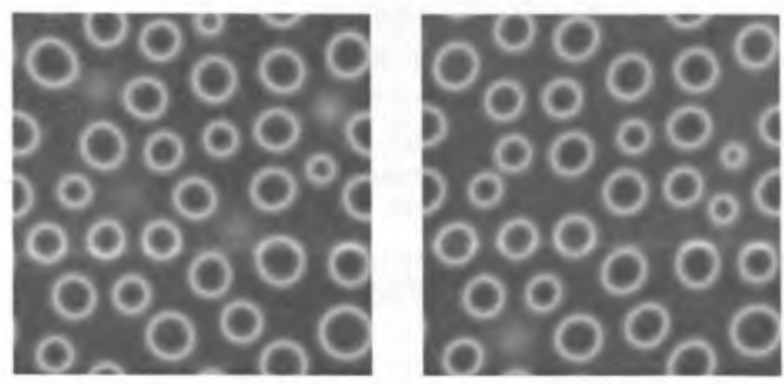

(d) $t_{80}=4$

FiG. 3. Paltern of two binodal separation processes at different times $t_{k}$ : left: results of the Cahn-Hilliard model simulation; right: results of the DM model simulation in the point $x_{1}$. The associated color bar is displayed in Figure 4. 


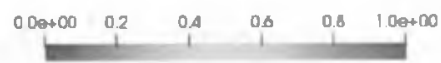

FIG. 4. Color bar giving values from 0 to 1 for the visualization of the onder pammeter $c$ in all subsequcnt figurcs.

A comparison of the two separation processes shows that the separation of the phases progresses slowlier in the DM-simulation in $x_{1}$ than in the Cahn-Hilliard sinnulation. This secms to be a gencral observation in macroscopic points close to the compression boundary $\Gamma_{\mathrm{g}}$. Even more significantly, the domains in the DM simulation are cllipsoidally shaped not only during fusion and they are aligned in the diagonal direction from the lower left corner to the upper right corner. The ellipsoid structure is already recognizable from the begimming and is most noticeable in the carly stages of the separation process, i.e., inmediatcly with the formation of the domains and can be clearly secu when looking at the emerging microstructure at times $t_{30}$ and $t_{40}$; cf. Figures 3(a) and 3(b).

The ellipsoidal structure is retained for at least a certain period of time, which can be seen particularly well at times $t_{40}, t_{60}$, and $t_{80}$ and in comparison to corresponding domains at the same times of the Cahn-Hilliard simulation. In the DM sinulation, there are domains which do not grow and remain cllipsoidally sliaped during the considered period.

Figure $\mathbf{5}$ shows patterns in different macroscopic points. The arrangement of the single plots rorresponds approximately to the position of the macroscopic points in $\Omega$ to which the pattcrns bclong; cf. Figure 2.

As can be seen from the figure, different alignment directions of the domains occur depending on the macroscopic position. The orientation of cllipsoidal donains is most obvious in points $x_{1}$ and $x_{5}$; sec Figures $5(\mathrm{a})$ and $5(\mathrm{c})$.

Generally, it can be observed that the oricntation direction of the domains in macroscopir points in the upper half of $\Omega$ is rather towards the lower left romer and the upper right corner (which corresponds to the points $x_{1}$ and $x_{2}$ ), wherens the domains in the macroscopic points of the lower half of $\Omega$ are rather oriented towards the upper left corner and the lower right corner (which corresponds to the points $x_{4}$ and $x_{5}$ ). In what follows, we refer to these two occurring directions, the two diagonals just described, as orientation diagonals for the alignuent of the patterns. While the shear stress in $x_{2}$ and $x_{4}$ is much smaller than in $x_{1}$ and $x_{5}$, respectively, it is negligilule in the point $x_{3}$, which lies in the middle along the vertical coordinate of $\Omega$. Accordingly, the diagonal orientation of the domains in Figures $5(\mathrm{~b})$ and $5(\mathrm{~d})$ is weak but still visible and nonexistent in $5(\mathrm{c})$. It can also be scen here (and in similar simulations not shown) that there seems to be a preferred direction in which domains merge with other domains. This direction corresponds to the direction of the respective oricutation angle of the cllipsoidal domains. These observations fully unect our initial expectations. The nuruscupic strain influences the phase sepatration. It has an effect on the shape and orientation of the domains and the dynamics of the separation process, e.g., mergiug of domains.

5.2. Spinodal phase separation. The influence of the locally different macroscopic strain l,ccomes more pronounced in the case of spinodal phase separation. Analogously to the binodal rase. we first compare the Cahu-Hilliard simulation with the simulation of the separation process in the macroscopic point $x_{1}$. The patterns 


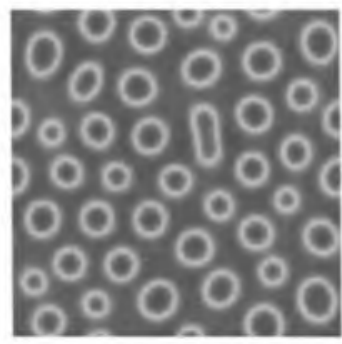

(c) Pattern at $x_{3}$.

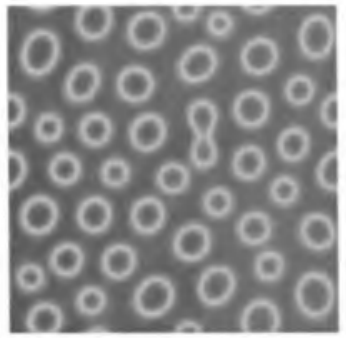

(b) Pattern at $x_{2}$.

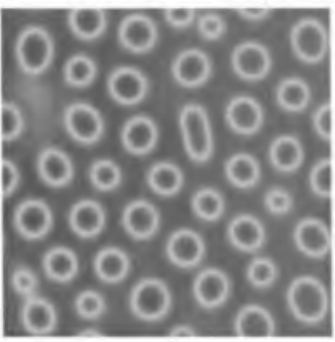

(d) Pattern at $x_{\mathrm{A}}$.

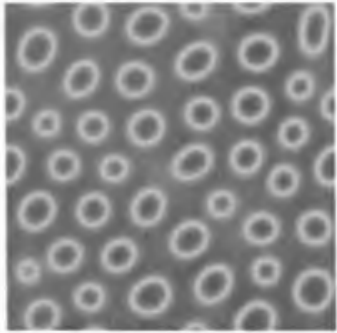

(a) Pattern at $x_{1}$.

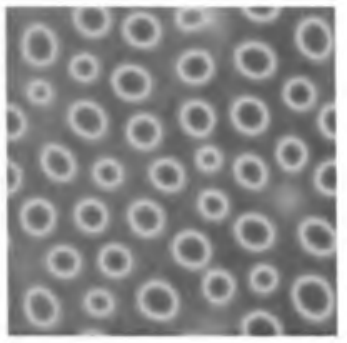

(c) Pattern at $x_{5}$.

FIG. 5. Pattern of the evolving microstructure in different macroscopic points at tame $t_{10}=2$ for binodal phase separation. The associaled color bar is displayed in Figure 4.

at different times can be seru in Figure 6. In the left column of the figure, the results of the Cahn-Hilliard simulation are shown while the solution of the DM simulation can be found in the right columu. Results next to each other are at the same times sperified underneath. For the comparison of the results from the two simulations, we lave chosen different points in time than for the corresponding comparison in the case of binodal separation. The reason for this is that the phase separation is faster here and the selected points in tine are adjusted accordingly.

The process of both simulations is roughly the same. In the beginning, the homogencous mixture separates and regions of pure phases are fonned, but in comparison to binodal phase separation, no circular or ellipsoidal domains but stripc-like structures are formed. As time progresses, the regions of pure phases grow, which corresponds to a reduction of the phase boundaries.

At time $t_{10}$, the phase separation is far from being complete in both cases. At this time, in contrast to the Cahn-Hilliard simulation, in the DM simulation a diagonal orientation of the mirrostructure is clearly visible already. This difference becomes more pronounced at time $t_{20}$. The plots of the DM simulation show the diagonal 
alignment of the pattern and there are structures which extend diagonally over alınost the entire domain. As time progresses, this preferential aligmment of the patterns also remains present during the growth of the pure phases as can be seen at tines $t_{40}$ and $t_{60}$. The structure of the pattern becones more and more similar to diagonal stripes as time progresses. A comparison of Figures $6(\mathrm{~b})$ and $6(\mathrm{~d})$ also slıows that the phase boundaries smoothen over time. The patterns of the Cahn-Ililliard simulation, on the other hand, still appear disordered and do not show any particular oricntation.

In Figure 7. results for different macroscopic points are shown. The selection of the macroscopic points and the arrangement of the single pictures is the same as in Figure 5 and corresponds approxinately to the position of the macroscopic points to which the patterns belong. In spinodal separation, the characteristics of the different orientations of the patterns are nore pronounced.

At first glance. it can be said that in all patterns shown in Figure 7 a certain orientation is noticcable in comparison to the corresponding results of the CahnHilliard simnlation; cf. Figure $6(d)$. The observation that we made in the binodal case during the discussion of Figure 5 also applies here, that is that the microstructure in those macroscopic points that is located in the upper or lower half of $\Omega$ is oriented along the respective diagonals. However, this orientation is seen much more strongly in the spinodal case. Similarly, the orientation of the patterns in $x_{1}$ and $x_{5}$ is strongly oriented to the diagoual while the patterns belonging to the points $x_{2}$ and $x_{4}$ are more vertical already. Located vertically centered in $\Omega$, the formation of the patten shown in Figure $7(c)$ is oriented vertically. This becomes particularly clear in comparison to the pattern of the pure Cahn-Hilliard simulation: ef. Figure 6(d).

5.3. Spinodal phase separation with other stiffnesses. Finally, we investigate the effects of a variation of the material stiffuess. For this purpuse, we corrsider a weaker and a stiffer material by taking Lamé coefficients multiplied by a factor of one third. i.c., $\lambda^{\alpha}=0.2 . \mu^{\alpha}=0.2$ and $\lambda^{\beta}=0.4, \mu^{\beta}=0.6$ and laking Lamé coefficients multiplicel by a factor of one and two thirds, i.c., $\lambda^{\alpha}=1, \mu^{\alpha}=1$ and $\lambda^{\beta}=2, \mu^{3}=3$, in comparison to the original choice given in (5.4) and (5.5) and used to produce the results shown so far.

The first row in Figure 8 shows the results of the DN sinulation at selected macroscopic points (specified underneath) with the decreased Lamé constants. In the sccond and the third row, the respective results of the DM simulation with the previously used Lamé constants given by (5.4) and (5.5) and with the increased Lamé constants at the sanc point in tinc can be scen. Figures $8(\mathrm{a}), 8(\mathrm{~d})$, and $8(\mathrm{~g})$ slow patterns in the vertically centered macroscopic point $x_{3}$ at time $t_{40}=2$. In the case of higher Lané constants, the vertical alignment of the pattern is unch more pronounced at this time. On the other hand, the results for the less stiff material given in Figure 8(a), slow less vertical aligument and are already similar to the results of a Cahn-Hilliard simulation: sce Figure 6(b). Figures 8(b), 8(c), and 8(h) show patterns in $x_{2}$, which is located in the upper half of $\Omega$. In the plot of the simulation with the values for elastically harder components, the oricutation of the patterns is stronger the stiffer the material. The same applies to the patterns shown in Figures $8(\mathrm{c}), 8(\mathrm{f})$, and $8(i)$ for the point $x_{1}$.

6. Summary and discussion. We considered the Cahn-Larché system in a multiscalc context. Assuming two distinct length scales, a microscopic and a macroscopic one. we obtained a Cahn-Larché system in terms of dimensionless quantities. where certain terms werc scaled with powers of the ratio of the length scales. For the case where the mechanics is assumed to take place on the macroscopic scale 

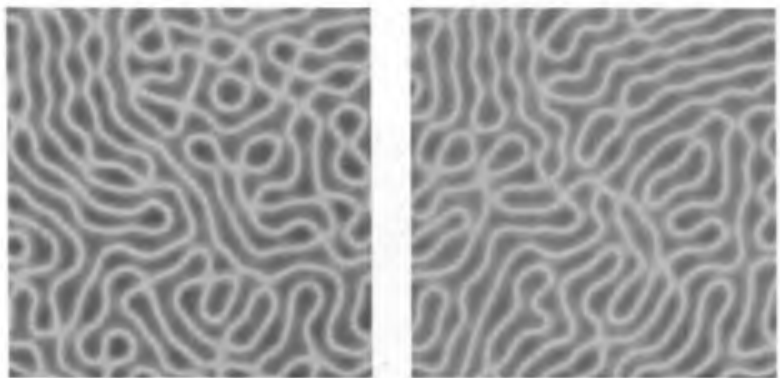

(a) $t_{10}=0.5$
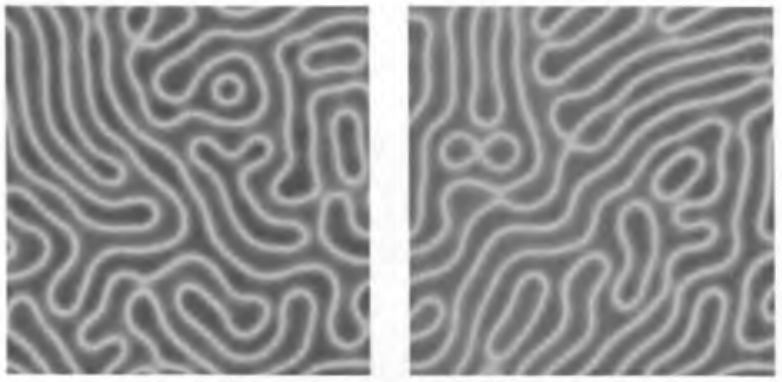

(b) $t_{20}=1$
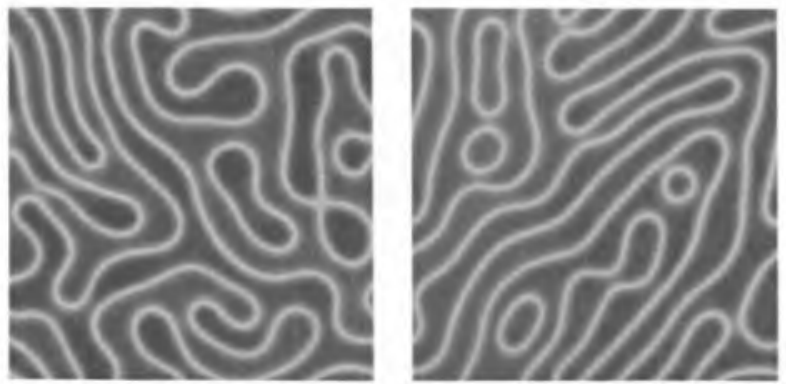

(c) $t_{10}=2$
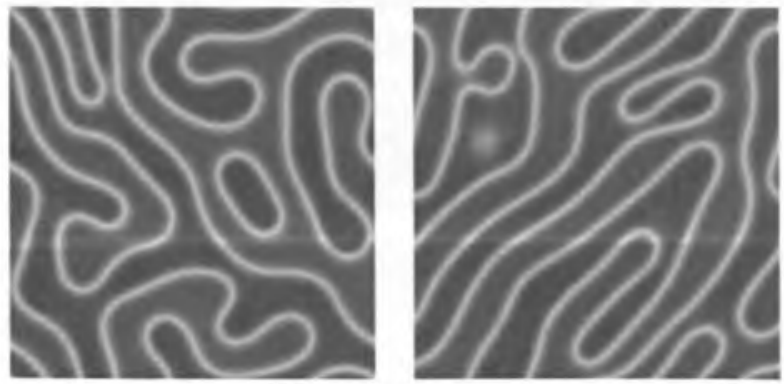

(d) $t_{80}=4$

FiG. 6. Paltern of two spinodal separation processes at different times $t_{k i}$ left: results of the Cahn-Hilliand model simulation; right: results of the DM model simulation in the macroscopic point $x_{1}$. The associated color bar is displayed in Figure 4. 


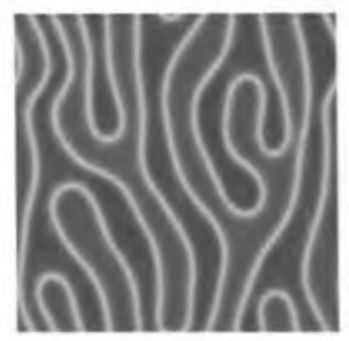

(c) Pattern at $x_{3}$.
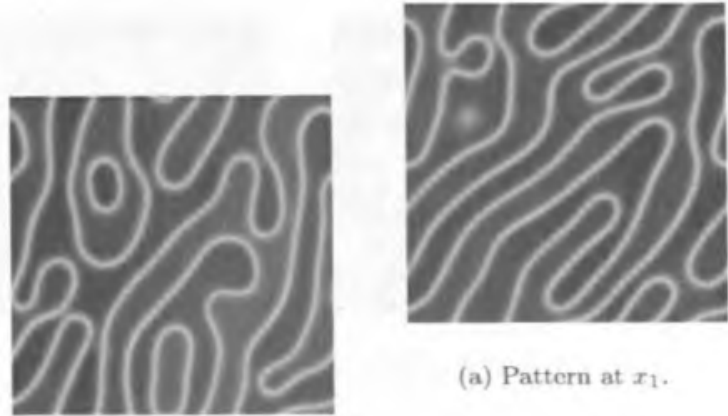

(a) Pattern at $x_{1}$. (b) Pattern at $x_{2}$.

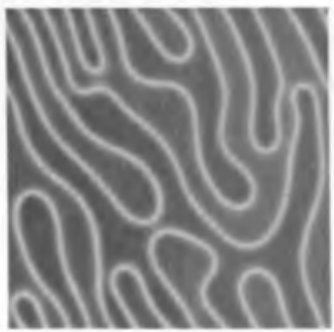

(d) Pattern at $\boldsymbol{x}_{1}$.

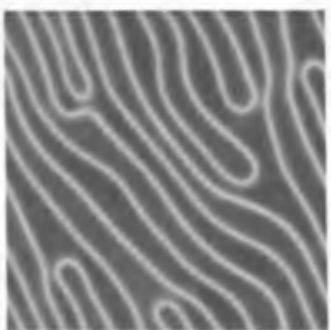

(c) Pattern at $x_{5}$.

Fig. 7. Pattern of the evolnng macrostructure at differenl macroscopic points at time $t_{80}=4$ for spinodal phase separation. The associated color bar is displayed in Figure 4.

and the separation process occurs on the microscopic scalc, which is the case in Langmuir-Blodgett film-balance experiments, we obtained an upscaled system of DM type using the method of asymptotic expansions in a periodic-homogenization setting.

Typical simulation results were presented for the homogenized DM model. As in a single-scale setting, where the separation process is described by the standard CalınLarché system, the influence of the mechanics on the patterns can be clearly seen during phase separation. In the DM model, this effect arises on the microscale and it is particularly cvident in the spinodal case. In the binodal case, ellipsoidal shapes were secn particularly clcarly during the early stage of plase separation. Over tinc, it could be observed that the ellipsoidal shapes relax into more circular shapes. In contrast, in the case of spinodal scparation, we found that the alignucnt of the patterns due to clastic stresses becomes clearer and stricter as time progresses. Furthermore, these effects appear more strongly with elastically harder matcrial paraneters. Nevertheless, in all cases, a certain alignment of the microstructure can be seen, which varies locally macroscopically and which is in line with our expectations regarding the model. 


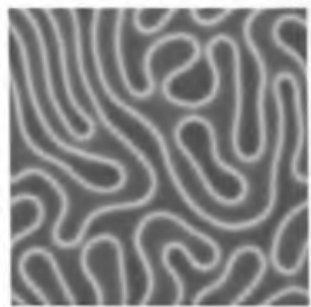

(a) Pattern at $x_{3}$

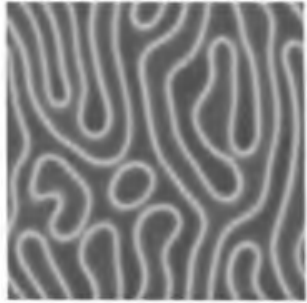

(d) Pattern at $x_{3}$

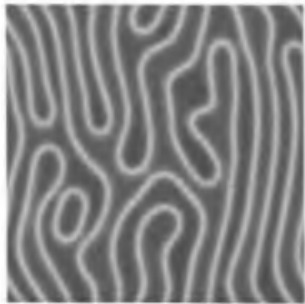

(g) Pattern at $x_{3}$

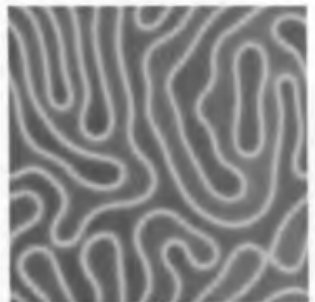

(b) Pattern at $x_{2}$

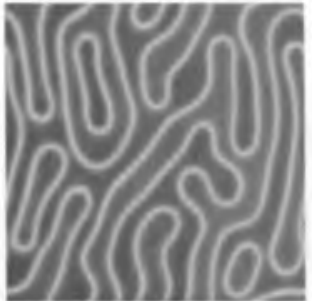

(e) Pattern at $x_{2}$

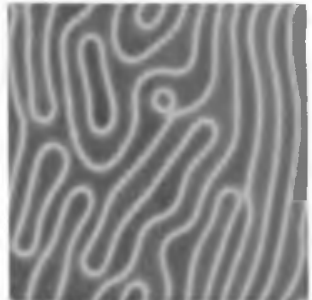

(h) Pattern at $x_{2}$

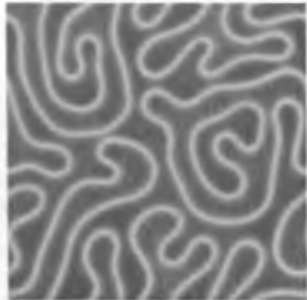

(c) Pattern at $x_{1}$

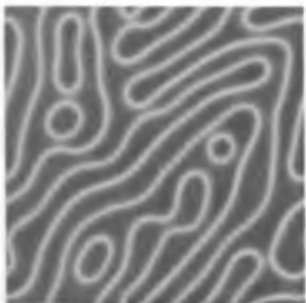

(f) Pattern at $x_{1}$

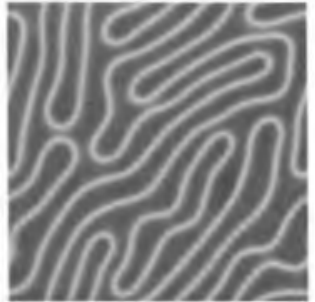

(i) Pattern at $x_{1}$

Fic. 8. Patlerns at different macroscopic points at time $t_{40}=2$ for elastically weaker (top row) and stiffer (bottom row) materials. The associated color bar is displayed in Figure 4.

Unfortunately, no quantitative measurements of the film-balance experiments. which motivated the developed multiscalc model, are available. A quantitative comparison with numerical simulations is required in the future to validate the limit model. Since the mathematics has been kept geueral, an application in thec dinensions is also feasiblc. For example, models of cvolving microstructures in phase-separating metal alloys under macroscopic mechanical stress could be considered in an analogous fashion. Furthermore, the upscaling method based on matched asymptotic expansions is mathematically licuristic. From an analytic point of vicw, passing to the limit in a mathematically rigorous way would be an important mathematical justification of the limit model. This poses a mumber of challenges, however, owing to the multiple nonlinearities and the $\epsilon^{2}$-scaling: 


\section{REFERENCES}

[1] T. Amugast, J. Dolglas Ju.. anu U. Horncig, Derivation of the double pormity model of single phase flow via homogenization theory, SIAM J. Math. Anal., 21 (1990), pp. 823-863.

[2] A. Bensolssan. J.-L. Lions, and G. Papanicolaul, Asymplotir analysis for periodic structures, North-Holland. Amsterdam, 1978.

[3] O. Boyahkin, S. Blikgeir. T. Franke, T. Fralinolz. R. H. W. Hoppe. S. Kirscilleir, K. Lindner. M. A. Petek. F. G. Strobl. Anv A. Wixforti, Trathport al interfaces in lipid membranes and enantiomer separatzon, in Transport Processes at Fluidic Interfaces. D. Bothe and A. Reusken, exls., Springer, Cham. Switzerland, 2017, pp. 489-530, https: //doi.org/10.1007/978-3-319-56602-3_17.

[4] S. Burger. T. Fralnilolz, C. Leilek. R. H. W. hoppl. A. Wixfortil. M. A. Peter. and T. FRANKE, Comparative study of the dynamics of lipid membrane phase decomposition in experiment and simulation, Langmuir, 29 (2013), pp. 7565-7570.

[5] D. Clohanescl and P. Donato, An Introduction to Homogenization. Oxford Lecture Ser. Math. Appl., Oxford University Press, Oxford, 1999.

[6] .J. EstIEl 13Y. Elastic inclusions and inhomogeneitics. in Progress in Solid Mechanics, Vol. 2, North Holland, Amsterdam. 1961, pp. 89-140.

[7] P. FlRatzl. O. Penrose. AND .1. Lemowitz, Modeling of phase separation in alloys writh coherent elostic misfit, J. Stat. Phys., 95 (1999), pp. 1429-1503.

[8] T. Fuackiolz, Transport at Interfaces in Lipid Membranes and Enantiomer Separation, PhD thesis, Universität. Augsburg, Augshurg, Germany, 2014. https://opus.bibliothek. uni-augsburg.dc/opus4/frontdoor/index/index/docld/3067.

[9] H. GarckE, On Mathematiral Models for Phose Separation in Elasfically Stressed Solids, Habilitation thesis, Universität Bonn, Bonn, Germany, 2000.

[10] M. Gudmani. M. Fluorka. T. BuURxholm AND T. HeiniblRg, Diffusion and partilioning of fluorescent lipid probes an phospholipid monolayers, Biophys. J., 96 (2009). pp. 4598-4609, ht tps://doi.org/10.1016/j.bpj.2009.01.06i3.

[11] U. Honnitag, ed., Homogenization and Pornus Media. Springer, New York, 1997.

[12] A. KHACIATLikyaN, Some questions conceming the theory of phase transformations in solids, Sov. Phys. Solid State, 8 (1967), pp. 21632168.

[13] K. KLOPFEK AND T. VANDERLICK, Isolherms of dipalmitoylphosphatidyltholine (DPPC) monolayers: Features revealed and features obscured. J. Colloid Interfacc Sci., 182 (1996), pp. 220-229.

[1.1] P. KRÏGER ANo M. Löscht, Molecular chiralily and domain shapes in lipid monolayers on aqueous surfaces, Phys. Rev. E(3), 62 (2000). pp. 7031-7043, https://doi.org/10.1103/ PhysRcrE.62.7031.

[15] T. KïppER AND X. MASBAL M, Simulations of particle growth and Ostwald, ripening via CahnHilliard equation, Acta Metall. Matcr., 42 (1994). pp. 1847-1858.

[16] F. LARCHÉ AND .J. CallN, The effect of self-stress on diffusion in solids, Acta Metall., 30 (1982), pp. 1835-1845.

[17] A. Logg. K.-A. Mardal, ANd G. V. Wells, eds., Automated Solution of Differential Equations by the Finite Element Method, Springer, Bcrlin, 2012.

[18] C. McConlogli and T. VANderlick, A close look at domain formation in DPPC monolayers, Langmuir, 13 (1997), pp. 7158-7164.

[19] S. A. MEje:, Two-Scale Models for Reactive. Transport and Evolving Microstructure, PhD thesis, Universität Bremen, Bremen, Germany, 2008.

[20] T. MERkLe, The Cahn-Lurché Systern: A Molel for Spinodal Decomposation in Eutectic Solder - Modelling, Analysis and Simulation, PhD thesis, Universität Stuttgart, Stuttgart, Germany, 2005.

[21] A. Miller and H. Mörwalo, Diffusion limited gruwth of crystallanc domains in phospholupid monolayers, J. Chem. Phys., 86 (1987), pp. 4258-4265, https://doi.org/10.1063/1.451886.

[22] A. Miranville, The Cahn-Hilliand Equation: Recent Advances and Applications, CBMS-NSF Regional Cunf. Ser. Appl. Math., SIAM, Philadelphia, 2019.

[23] H. MöıwAlo, Phospholıpid monolayers, in Structure and Dynamics of Membrancs. R. Lipowsky and E. Sackmann, eds., Handb. Biol. Phys. 1, North-Holland. Amsterdam. 1995, pp. 161-211, https://doi.org/10.1016/S1383-8121(06)80021-7.

[24] T. Mlira, General theory of eigenstrains, in Micromcchanics of Defects in Solids, Springer, 1987, Dordrecht. The Netherlands, pp. 1-73. https://doi.org/10.1007/978-94-009-3:489-4_ 1. 
[25] K. NaG. C. Botand. N. Rici, AND K. KEolgh, Eptfluonescence mictoscopic observation of monolayers of dipalmitoylphosphatidylcholine: dependence of domain size on compression rates, Biochem. Biophys. Acta., 1068 (1991), pp. 157-160.

[26] M. Panfilov, Macroscale Models of Flow Through Highly Heterogeneous Porous Media. Kluwer Academic, Dordrecht, The Netherlands, 20К.

[27] M. A. Petek AND M. Bölim, Sralings in homogenusation of reaction, diffusion and interfacial exchange in a two-phase medium, in Procedings of Equadiff 11, Intenational Conference on Differential Equations. M. Fila. A. Handlovicova, K. Mikula, M. Medved, P. Quittner. and D. Sevcovic, eds., Comenius University, Bratislava, Slovakia, 2005, pp. 369-37t, http: //www.jaln.fuph.umilıask/equadiff/htmls/_procecdings/_peter/peter.pdf.

[28] M. A. PETEK AND M. BöliM, Different choises of scalıng in homogenzation of diffusion and interfacial exchange in a porous medium, Math. Methods Appl. Sci., 31 (2008), pp. 12571282.

[29] M. A. PETER AND M. BöluM, Multiscale modeling of chemical degradalion mechanisms in porous media uith evolving microstructure, Multiscale Modcl. Simul., 7 (2009), pp. 16431668, https://doi.org/10.1137/070706410.

[30] E. SÁxchez-PAlencia, Non-homogeneous media and vibration theory, Lecture Notes in Phys. 127, Springer, Berlin, 1980.

[31] R. E. Showalter, Diffusion models unth microstructure, Transp. Porous Mcdia, 6 (1991), pp. $567-580$, https://doi.org/10.1007/BF00137850.

[32] R. E. Showaltek, Distribuled mictostructure models of pormus media, in Flow in Porous Media: Proccedings of the Oberwolfach Conference, June 21-27, 1992, J. Douglas Jr. and U. Hornung, eds., Birkhäuser, Basel. 1993, pp. 155-163, https://doi.org/10.1007/ 978-3-0348-8564-5_14.

[33] Sigmla-AldRICH, Data of DPPC, 2019, https://www.sigmaaldrich.com/catalog/product/ avanti/850355c.

[34] D. STEPPICH, Kopplung von mechanischen und thermodynamischen Eigenschaften von Phospholipidmembranen in der Nähe von Phaserumwandlungen - Bedeutung für Anwendungen und Biologie, diploma thesis, Universität Augsburg, Augsburg, Germany, 2005.

[35] P. Toimil. X. Pirieto. .J. Miñones, and F'. Sarmento, A compamtive study of $f$ $D P P C / D P P C$ mixed monolayers. infiuence of subphase temperature on $f-D P P C$ and DPPC monolayers, Phys. Chem. Chem. Phys., 12 (2010), pp. 13323-13332, https://doi. org/10.1039/c0cp00506a.

[36] U. Weikakd, Numerusche Lösungen der Cahn-Hallard-Gleschung und der Cahn-LarchéGleichung, Ph.D thesis, Rheinische Fricdrich-Wilhelms-Universität Bonn, Bonn, Germany, 2002. 